Temperature and nutrients drive complex eco-phenotypic dynamics in a microbial food web

3

\author{
Ze-Yi Han ${ }^{1}$, Daniel J. Wieczynski ${ }^{1}$, Andrea Yammine ${ }^{1}$, Jean P. Gibert ${ }^{1}$ \\ ${ }^{1}$ Department of Biology, Duke University, Durham, North Carolina, USA
}

Statement of authorship: ZH and JPG conceived the study; ZH and JPG designed the experimental work. ZH collected the data with support from AY. ZH analyzed the data with support from DJW and JPG; ZH wrote the first version of the manuscript; all authors contributed substantially to subsequent versions.

Data accessibility statement: All annotated code and data is available at our dedicated repository (https://github.com/ZeYiHan/Nut_Temp_Pheno_Eco).

Running title: Consequences of thermal asymmetries

Keywords: Global warming, Food-web temperature responses, Eutrophication, Anthropogenic impacts, Communities, Trait-based Ecology

\section{Type: Letter}

Number of words: abstract (150 words), main text (3983 words), boxes ( 0 words)

\section{Number of figures: 5}

Number of references: 48

Number of tables: 0

Number of boxes: 0

To whom correspondence should be addressed: Ze-Yi Han, Department of Biology, Duke

University, Durham, NC, USA, 402-405-3888, zeyihanw@gmail.com

37

(1)


41 ABSTRACT

42 Anthropogenic increases in temperature and nutrient loads will likely impact food web structure

43 and stability. Though their independent effects have been well studied, their joint effects-

44 particularly on coupled ecological and phenotypic dynamics-remain poorly understood. Here

45 we experimentally manipulated temperature and nutrient levels in microbial food webs and used

46 time-series analysis to quantify the strength and causality of reciprocal effects between

47 ecological and phenotypic dynamics across species. We found that i) temperature and nutrients

48 have more complex effects on ecological dynamics at higher trophic levels, ii) temperature and

49 nutrients interact antagonistically to shift the relative strength of top-down vs. bottom-up control,

50 iii) phenotypic dynamics have stronger impacts on ecological dynamics than vice versa

51 (especially at higher temperature), and iv) rapid phenotypic change mediates observed ecological

52 responses to changes in temperature and nutrients. Our results expose how feedbacks between

53 ecological and phenotypic dynamics mediate food web responses to environmental change. 


\section{INTRODUCTION}

65 Understanding how rapid global climate change (GCC) will affect the structure and dynamics of

66 communities is a pressing goal of ecology (Karl 2003). Increasing temperatures associated with

67 GCC influence the metabolism of individuals (Clarke 2006), which strengthens species

68 interactions (Barton et al. 2009; O'Connor 2009; Gounand et al. 2016), alters community

69 structure (Bartley et al. 2019; Gibert 2019; Gauzens et al. 2020), and ecosystem function

70 (Dossena et al. 2012). Additionally, increasing nutrient loads from agricultural runoffs can result

71 in eutrophication and destabilize natural communities (Rosenzweig 1971; Fussmann 2000;

72 Hautier et al. 2014), often leading to species loss (Hautier et al. 2009). Warming and

73 eutrophication may therefore independently and jointly impact food web structure and stability

74 (Malcolm et al. 2006; Tabi et al. 2019). Counterintuitively, however, simultaneous increases in

75 temperature and nutrient load can produce outcomes that are qualitatively different from the

76 combined negative effects of each variable on its own (Binzer et al. 2012, 2016). These non-

77 additive (interactive) outcomes are still poorly understood, but central to honing our predictions

78 about GCC impacts on food web structure and dynamics in a highly anthropogenized world.

80 The mechanisms through which warming and increasing nutrient loads independently influence

81 food webs are relatively well understood (McClelland \& Valiela 1998; Carlier et al. 2008; Gibert

82 2019), specifically regarding their impacts on the relative strength of bottom-up and top-down

83 effects (Binzer et al. 2012, 2016; Shurin et al. 2012). For example, warming can increase

84 predation pressure (Kratina et al. 2012), thus decreasing resource biomass, while increasing the

85 proportion of top predators (Vasseur \& McCann 2005; O'Connor 2009; Shurin et al. 2012).

86 Alternatively, warming can increase metabolic demands while reducing conversion efficiency 
87 (Barneche et al. 2021), leading to predator starvation at high temperatures, loss of top predators,

88 and reduced food chain length (Petchey et al. 1999)Unlike warming, increasing nutrient loads

89 tend to increase bottom-up effects, often resulting in unstable dynamics and species loss (i.e.,

90 paradox of enrichment, Rip and McCann, 2011; Rosenzweig, 1971). Increasing nutrient loads

91 can alter consumer-resources interactions, sometimes resulting in top-heavy, unstable food webs

92 (Rip \& McCann 2011). Eutrophication resulting from increasing nutrient loads can also change

93 consumer trophic position, leading to changes in species interactions and food web structure (Lee

94 et al. 2021).

95

96 While warming and nutrients can independently influence food webs, they can also have non-

97 additive (interactive) effects when acting together (Binzer et al. 2012, 2016; Tabi et al. 2019).

98 For example, at low temperatures, small temperature increases can stabilize nutrient-induced

99 instabilities (Binzer et al. 2012, 2016), although temperature increases are themselves considered

100 destabilizing because they strengthen consumer-resource interactions (Barton et al. 2009). At

101 high temperatures, increasing nutrient loads can counteract warming-induced consumer

102 starvation by increasing carrying capacity and predator attack rates (Binzer et al. 2012).

103 Warming also weakens nutrient-induced increases in community biomass (negative interactive

104 effects, Tabi et al., 2019), in turn influencing food web structure (Sentis et al. 2014), species

105 richness, and community composition (Wang et al. 2016).

106

107 Temperature and nutrients can both determine the physiological and morphological traits of

108 organisms (Rosenblatt \& Schmitz 2016; Tabi et al. 2019). For example, higher temperatures

109 often result in smaller sizes (Atkinson 1995; Atkinson et al. 2003), while nutrient enrichment 
110 leads to larger organisms (Irwin et al. 2006; Marañón et al. 2013). Additionally, temperature and

111 nutrients can interactively affect body size, where body size may increase with nutrients at low

112 temperature but decrease at high temperature (Tabi et al. 2019). Although body size is often

113 considered a response variable, its strong influences on population growth (Fenchel 1974;

114 Savage et al. 2004) and species interactions (Ferenc \& Sheppard 2020) mean that rapid

115 phenotypic responses to temperature, nutrients, or both, may have consequences for food web

116 structure and dynamics in warmer climates (Brose et al. 2012; Bernhardt et al. 2018; Wieczynski

117 et al. 2021). However, whether body size only responds to environmental conditions, whether

118 rapid changes in body size influences how environmental conditions affect food webs, or

119 whether shifts in body size in a species can influence body size responses to environmental

120 conditions in the species they interact with, are not well understood.

122 Here, we study how temperature and nutrients influence the feedbacks between ecological and 123 body size dynamics in a tractable microbial food web by first describing observed food web and

124 body size responses to temperature and nutrients across trophic levels, then studying the

125 mechanisms of response. Specifically, we ask: 1) do temperature and nutrients independently or

126 interactively influence ecological dynamics within microbial food webs?, 2) do these effects alter

127 the relative importance of top-down vs. bottom-up control?, 3) do these effects vary across

128 trophic levels?, and 4) is body size only responding to temperature and nutrients, as suggested

129 elsewhere (e.g., Binzer et al 2016, Tabi et al 2019), or does that response play a role in

130 determining how the food web itself responds to environmental change. To address these

131 questions, we manipulate nutrient inputs and temperature in a microbial food web composed of

132 bacteria, a bacterivorous protist (Tetrahymena pyriformis), and an omnivorous top predator 
133 (Euplotes sp.), then track the changes in population densities and body sizes of over time. We

134 use state-of-the-art time-series analysis to evaluate the relative strength of top-down vs. bottom-

135 up processes across trophic levels, as well as whether and how the observed ecological and

136 phenotypic dynamics influence one another. Our results reveal complex temperature and nutrient

137 effects on food web dynamics that vary predictably across trophic levels by altering the relative

138 strength of trait-mediated bottom-up and top-down effects.

141 METHODS

142 Culture care

143 Euplotes sp. and Tetrahymena pyriformis stock cultures were acquired from Carolina Biological

144 Supply (Burlington, NC, USA). Both species were kept in autoclaved liquid protist medium

145 (Carolina Biological Supply), supplemented with one autoclaved wheat seed as a carbon source

146 (Altermatt et al 2014). Protists were fed a mixture of pond bacteria collected from an ephemeral

147 pond at Duke Forest (Wilbur/Gate 9 pond, Lat 36.013914, -Long 78.979720). We maintained all

148 cultures on a 16:8 light:dark cycle at $22^{\circ} \mathrm{C}$ and $65 \%$ humidity in AL-22L2 Percival growth

149 chambers (Perry, IA, USA).

151 Experimental design

152 Microcosms were set up in autoclaved $250 \mathrm{~mL}$ borosilicate jars filled with $200 \mathrm{~mL}$ of protist

153 media. We manipulated temperature and nutrient loads by imposing two temperature levels

$154\left(22^{\circ} \mathrm{C} / 25^{\circ} \mathrm{C}\right)$ and two nutrient levels (normal protist media concentration plus one wheat seed -

155 i.e., high nutrients-, or half concentration plus half a wheat seed, -i.e., low nutrients) in a 
156 factorial design with 4 treatments and 6 replicates per treatment. Day/night cycle and humidity

157 levels mimicked rearing conditions and were kept constant. We inoculated the bacterial

158 communities from $2 \mathrm{ml}$ of stock culture, the same used to rear the protists. The intermediate

159 consumer species, T. pyriformis, was introduced at a starting density of $37 \mathrm{ind} / \mathrm{ml}$, and the larger

160 protists species, Euplotes sp., at a starting density of $0.24 \mathrm{ind} / \mathrm{ml}$ in all microcosms. We recorded

161 the density of both protist species through fluid imaging (FlowCam; Yokogawa Fluid Imaging

162 Technologies, Portland, ME, USA), Monday through Friday, for 16 days. Fluid imaging

163 generates individual cell raster images that were used to quantify changes in protist size

164 (measured as cell area, in $\mu^{2}$ ) over time. Bacteria density was quantified as optical density at a

165 wavelength of 600nm (OD600), using a BioTEK Epoch-2 microplate spectrophotometer

166 (Winooski, VT, USA).

168 Data analysis

169 To test for possible effects of temperature and nutrients on ecological dynamics, we fitted

170 Generalized Additive Mixed Models (GAMM) to time series of species density (OD600 for

171 bacteria) and protist body size, across all treatments, using the 'mgcv' package (version 1.8-

172 31,(Wood 2011)) in R (version 4.0.2, R Core Team 2020). To control for temporal

173 autocorrelation, we used an Autoregressive Moving Average (ARMA) correlation structure in

174 the GAMM models using the 'nlme' R package (version 3.1-148). We compared models with

175 additive and/or interactive effects temperature and nutrients effects, as well as different ARMA

176 correlation structures, using AICc (Appendix I Table S1-2). We discarded T. pyriformis

177 phenotypic data from days on which less than 10 individuals were measured after populations

178 collapsed. 


\section{Characterization of ecological dynamics}

181 To assess environmental effects on microcosm food web dynamics, we characterized multiple

182 aspects of the population dynamics of bacteria and protists and their responses to environmental

183 conditions. Specifically, we quantified: 1$)$ initial growth rate $\left(\right.$ day $\left.^{-1}\right)$ as $[\ln (\mathrm{Nf})-$

$184 \ln (\mathrm{Ni})$ ]/time for early-dynamics data, (up to day 1 for $T$. pyriformis \& bacteria and day 8 for

185 Euplotes $s p$., due to much slower growth), 2) maximum density (individual/ml for protists and

$186 \mathrm{OD}_{600}$ for bacteria), using the highest average daily population density across replicates but

187 within treatments for protists, and the top three observed OD600 values for each replicate from

188 each treatment for bacteria because their population dynamics remained relatively stable, 3) the

189 coefficient of variation $(\mathrm{CV}=$ standard deviation/mean $)$ of the temporal population dynamics

190 within treatments (typically used as a measure of stability (McCann 2012)), 4) the time to

191 population collapse in days (only $T$. pyriformis), and 5) the time to population peak in days (only

192 Euplotes sp.).

193

194 Quantifying top-down/bottom-up effects and feedbacks between ecological and body size

195 dynamics

196 To understand the mechanisms through which temperature and nutrients affected the observed

197 food web dynamics, we quantified the reciprocal effects of ecological and body size dynamics

198 using Convergent Cross Mapping (CCM) from the R package 'rEDM' (version 1.5.0; Park et al

199 2020). CCM quantifies the strength of causation of one dynamical variable onto another by

200 measuring the extent to which the time series of one variable X can reliably estimate the state of

201 another variable $\mathrm{Y}$ in a future time step (Sugihara et al. 2012). The higher the ability of $\mathrm{X}$ to 
202 predict $\mathrm{Y}$, the higher the causal effect of $\mathrm{Y}$ on $\mathrm{X}$. Meanwhile, a variable $\mathrm{Y}$ that does not

203 influence a variable X cannot be predicted from the dynamics of X (Sugihara et al. 2012). This

204 procedure yields a 'predictability' value that can be interpreted as a correlation coefficient

205 between past and future states of the two dynamical variables (Sugihara et al. 2012). Whether

206 there is a cause-effect relationship between two dynamical variables, as opposed to simple

207 correlation, further depends on whether the average predictability value increases with library

208 size (i.e., number of data points taken into account to run the analysis, Sugihara et al., 2012). We

209 took this into account to infer causation in our data (see Appendix II Fig. S1-14).

211 CCM is often applied to relatively long time series (Sugihara et al. 2012). However, the

212 dynamics in our system occurred much too fast (Fig. 1), precluding us from collecting longer

213 time series. To resolve this issue, we used CCM on the GAMM model predictions of changes in

214 density (for all species) and body size (for protists) with time, for each treatment. These GAMM

215 model predictions considered the effects of each treatment as a whole-i.e., each combination of

216 nutrient and temperature levels - because they more closely reproduced the observed dynamics

217 (Appendix I Table S1). We refer to these GAMM predictions as 'time-series' in the context of

218 CCM analyses. Using CCM on the density time series, we calculated the predictability of

219 changes in prey density from changes predator density (i.e., bottom-up control, as predator

220 density predicting prey density means changes in prey density causes changes in predator

221 densities, Sugihara et al., 2012) and the predictability of changes in predator density from

222 changes in prey (i.e., top-down control). We dubbed these "eco-eco" effects, for simplicity. To

223 assess feedbacks between body size and ecological dynamics, we calculated the predictability of

224 changes in protist body size from changes in species density (i.e., causal effects of changes in 
225 body size on densities, or 'pheno-eco' effects), and vice versa ('eco-pheno' effects). Last, we 226 calculated the predictability of changes in predator and prey body sizes on each other ("pheno-

227 pheno’ effects).

\section{RESULTS}

\section{General Ecological and Phenotypic dynamics}

232 Overall, bacterial density increased to carrying capacity early (Fig. 1a, light grey), T. pyriformis 233 increased rapidly, then decreased (Fig. 1a, dark grey), while Euplotes sp. increased almost

234 monotonically to carrying capacity (Fig. 1a, black), ultimately resulting in a two-species state.

235 Temperature and nutrients affected all three species and led to significantly different dynamics

236 across treatments (Fig. 1b-d, Appendix I Table S2). The body sizes of both protists changed

237 rapidly over time (Fig. 1e, f) and responded to both temperature and nutrients (Fig. 1e, f, 238 Appendix I Table S2).

240 Temperature and nutrient effects change across trophic levels in systematic ways

241 Temperature significantly affected a larger number of dynamical descriptors than nutrients (Fig.

242 2, 3). Individual temperature effects were more frequent in the upper trophic levels than in

243 bacteria (Fig. 3), as were interactions between temperature and nutrients (Fig. 3). In particular,

244 higher temperature alone i) strongly increased initial growth rate in Tetrahymena $(\mathrm{T}=0.50025, \mathrm{p}$

$245=1.16 \times 10^{-6}$, Fig. $\left.2 \mathrm{~b}\right)$, ii) decreased the maximum density of bacteria and T. pyriformis $\left(\mathrm{T}_{\mathrm{B}}=-\right.$

$246 \quad 0.004917, \mathrm{p}=0.00419 ; \mathrm{T}_{\mathrm{T}}=-975.3, \mathrm{p}<0.0155 ;$ Fig. 2d-e) but increased that of Euplotes sp. $\left(\mathrm{T}_{\mathrm{E}}\right.$

$247=39.83, \mathrm{p}=0.02668$, Fig. 2f), iii) increased the variability in $T$. pyriformis density (i.e. $\mathrm{CV}, \mathrm{T}=$ 
$0.20033, \mathrm{p}=0.00554$, Fig. 3h) but decreased that of Euplotes $s p .(\mathrm{T}=-0.07689, \mathrm{p}=0.01692$,

Fig. 3i), iv) accelerated the time to collapse in T. pyriformis $\left(\mathrm{T}=-2.7576, \mathrm{p}=1.59 \times 10^{-5}\right.$; Fig. $\left.2 \mathrm{j}\right)$,

250 and v) accelerated the time to peak density in Euplotes sp. $(\mathrm{T}=-2.1667, \mathrm{p}=0.0196$; Fig. 2k).

251 Higher nutrients alone i) decreased the initial growth rate of Euplotes $s p .(\mathrm{N}=-0.087, \mathrm{p}=$

$2525.02 \times 10^{-4}$ Fig. $\left.2 c\right)$, ii) increased maximum density of all species $\left(\mathrm{N}_{\mathrm{B}}=0.012717, \mathrm{p}=2.03 \times 10^{-}\right.$

$253{ }^{10} ; \mathrm{N}_{\mathrm{T}}=1002.2, \mathrm{p}=0.0132 ; \mathrm{N}_{\mathrm{E}}=60.67, \mathrm{p}=0.00157$, Fig. 2d-f), and iii) increased the $\mathrm{CV}$ of

254 bacteria and Euplotes sp. $\left(\mathrm{N}_{\mathrm{B}}=0.17521, \mathrm{p}=8.54 \times 10^{-5}\right.$, Fig. $2 \mathrm{~g} ; \mathrm{N}_{\mathrm{E}}=0.15853, \mathrm{p}=2.94 \times 10^{-5}$,

255 Fig. 2i) while decreasing that of T. pyriformis $\left(\mathrm{N}_{\mathrm{T}}=-0.18727, \mathrm{p}=0.00875\right.$, Fig. $\left.2 \mathrm{~h}\right)$.

256 Interestingly, increased temperature and nutrients had opposite effects on the CV of T. pyriformis

257 and Euplotes sp., indicating antagonistic additive effects of temperature and nutrients on food

258 web stability.

260 Temperature and nutrients had complex interactive effects on ecological dynamics of both protist

261 consumers. Nutrients had a negative effect on the initial growth rate of Euplotes sp. at low

262 temperature, but a positive effect at high temperature $(\mathrm{N} \times \mathrm{T}=0.12621, \mathrm{p}=0.000391$; Fig. 2c $)$.

263 The maximum density of $T$. pyriformis increased with nutrients, but this effect was stronger at

264 high temperature $(\mathrm{N} \times \mathrm{T}=1526.7, \mathrm{p}=0.0083$, Fig. 2e $)$. Nutrients also had i) a negative effect on

265 T. pyriformis population variability $(\mathrm{CV})$ at low temperature but no effect at high temperature

$266(\mathrm{~N} \times \mathrm{T}=0.19492, \mathrm{p}=0.04500$, Fig. 2h), and ii) a positive effect on Euplotes sp. $\mathrm{CV}$ that was

267 stronger at low temperature $(\mathrm{N} \times \mathrm{T}=-0.12045, \mathrm{p}=0.00913$, Fig. 2i). Additional model stats can

268 be found in Appendix I Table S3. Taken together, these results imply that temperature and

269 nutrients individually and interactively influence population dynamics at all trophic levels, with

270 more of both types of effects occurring at higher trophic levels (Fig. 3). 
Temperature and nutrients influence top-down and bottom-up effects

273 Using CCM analysis, we estimated changes in the relative strength of bottom-up and top-down

274 ecological effects across treatments (Fig. 4a). In general, top-down and bottom-up effects

275 between the top predators were the strongest (Fig. 4a, yellow). Top-down effects involving

276 bacteria were the most strongly influenced by temperature and nutrients (Fig.4a, green \& purple

277 dashed lines). Importantly, temperature and nutrients showed interactive effects on top-down and

278 bottom-up control. Most notably, top-down control by both protists on bacteria decreased with

279 nutrients at low temperature but increased with nutrients at high temperatures (Fig.4a, open dots

280 and dashed lines).

282 The most dominant species interactions in the overall food web also changed according to shifts

283 in the strength of top-down and bottom-up control between species pairs (Figure 5a). To be

284 conservative and increase the chance of picking up biologically relevant effects, we only present

285 effects whose CCM predictability value was above 0.75 in Figure 5 (Appendix III Table S1).

286 Specifically, increasing nutrients weakened top-down control from T. pyriformis to bacteria at

287 low temperatures (Fig. 5a left panel, dashed purple line), but strengthened top-down control from

288 both protists to bacteria at high temperatures (Fig. 5a right panel, dashed lines). In other words,

289 top-down effects were strongest when temperature and nutrients were both low, or both high,

290 suggesting that shifts in the relative importance of top-down and bottom-up effects across trophic

291 levels explain some effects of temperature and nutrients on the overall food web.

293 Temperature and nutrients alter the reciprocal effects of ecological and phenotypic dynamics 
294 Protist size not only responded to temperature and nutrients, but also played an important role in

295 determining the effects of temperature and nutrients on ecological dynamics (Fig. 4b-c). On

296 average, higher temperature generally led to stronger eco-pheno effects (mean $\mathrm{CCM}$ at $22^{\circ} \mathrm{C}=$

297 0.55, mean CCM at $25^{\circ} \mathrm{C}=0.66$, Fig. 4b, Appendix II Table S1) and pheno-eco interactions (size

298 driving ecological dynamics, mean $\mathrm{CCM}$ at $22^{\circ} \mathrm{C}=0.71$, mean $\mathrm{CCM}$ at $25^{\circ} \mathrm{C}=0.79$, Fig. $4 \mathrm{c}$,

299 Appendix II Table S1), indicating stronger coupling between ecological and phenotypic

300 dynamics at higher temperatures. Pheno-eco effects were generally stronger (14 strong effects,

301 i.e., CCM predictability > 0.75, Fig. 4b) than eco-pheno effects (11 strong effects, Fig. 4c),

302 suggesting stronger effects of body size on ecological dynamics than the other way around. For

303 example, Euplotes sp. size had stronger effects on its own population dynamics than the opposite

304 (Fig. 4b-c, black dotted lines).

306 Both eco-pheno and pheno-eco effects generally responded interactively to changes in

307 temperature and nutrients. For instance, T. pyriformis eco-pheno effects on Euplotes. sp (yellow

308 solid lines in Fig.4b) decreased with nutrients at low temperature but remained relatively

309 unchanged at high temperature. Also, nutrients increased the effect of T. pyriformis size on

310 bacterial dynamics and this pattern was more exaggerated at higher temperature (Fig. 4c, dashed

311 purple lines). Meanwhile, nutrients decreased the effects of Euplotes sp. size on bacteria

312 dynamics at low temperature and this pattern was reversed at high temperature (Fig. 4c, dashed

313 green lines). In 22 out of the 25 eco-pheno or pheno-eco interactions, we found strong support

314 for causation (Appendix II Fig. S1-14). 
316 Pheno-pheno effects between protists (Fig. 4d) were relatively weaker than pheno-eco or eco-

317 pheno effects. In general T. pyriformis size more strongly affected Euplotes sp. size than the

318 other way around (Fig. 4d). Additionally, temperature generally increased body size effects

319 between protists and we observed the strongest phenotypic coupling between protists when

320 temperature and nutrients were both high (Fig. 4d), indicating that higher temperatures may

321 increase the role of phenotypic dynamics in consumer-resource interactions.

323 Overall, these results indicate that the effect of temperature and nutrients on ecological dynamics

324 may be mediated by changes in body size (Fig. 5). Temperature and nutrients showed negative

325 interactions on strong eco-eco effects (CCM predictability $>0.75)$, leading to stronger eco-eco

326 effects when temperature and nutrients were both low or both high (Fig. 5a). However, the

327 number of strong causal effects between ecological and phenotypic dynamics increased with

328 temperature but were weakened by nutrients (Fig. 5b).

\section{DISCUSSION}

332 Our results reveal complex but systematic effects of temperature and nutrients on the ecological

333 and phenotypic dynamics of a microbial food web. We show that temperature and nutrients can

334 each independently influence different aspects of food web dynamics (Fig. 1, 2), while their joint

335 effects get increasingly complex at higher trophic levels (Fig. 2, 3). We also found that changes

336 in the relative strength of top-down and bottom-up effects likely drive observed responses to

337 temperature and nutrients (Fig. 4, 5), and that rapid changes in body size mediate these effects

338 (Fig. 4, 5). These results suggest that body size not only responds to shifts in environmental 
conditions, but also plays a role in determining ecological responses to such shifts. Evaluating

340 feedbacks between ecological and phenotypic dynamics is therefore integral to understanding

341 food web responses to environmental change.

Increasingly complex effects of temperature and nutrients at higher trophic levels

344 Our results reveal the pervasive effects of temperature and nutrients across all species, but these

345 effects are more numerous and increasingly complex (temperature-nutrient interactions) at higher

346 trophic levels (Fig. 2, 3). The effects of temperature and nutrients on dietary preference, species

347 interactions ${ }_{2}$ and foraging behavior may help explain why these interactive effects may be more

348 likely among consumer species (Fig. 1d; Fig. 2c).

350 Because energy enters at the bottom of a food web, basal species are more likely to be influenced

351 by direct effects of nutrients while species at higher trophic levels may be more strongly affected

352 by temperature (Petchey et al. 1999; Voigt et al. 2003)8/12/21 11:03:00 AM. As energy flows

353 from the basal species to the consumers, the temperature and nutrient effects that act on basal

354 species could be integrated by consumers, leading to additional indirect temperature and nutrient

355 effects on consumers. Additionally, these effects may be exaggerated in omnivores due to the

356 complex nature of their trophic interactions. As temperature and nutrients change species

357 interactions and foraging behaviors (Sentis et al. 2014), omnivorous consumers may shift diets

358 between basal and intermediate resources (Sentis et al. 2014), altering the strength/prevalence of

359 competitive vs. predatory interactions (Vandermeer 2006), thus rewiring food webs (Bartley et

360 al. 2019). Taken together, this may indicate why the joint effects of temperature and nutrients

361 may be more complex at higher trophic levels in food webs with higher prevalence of omnivory. 
Top-down control, bottom-up control, and food web stability

364 Consistent with our results (Fig. 2d), theory predicts that higher nutrient loads should increase

365 energy flux in food webs, increasing basal species density (Rip \& McCann 2011; Shurin et al.

366 2012). Theory also predicts that increasing energy flux can destabilize population

367 oscillations(Rosenzweig 1971; Rip \& McCann 2011), but increasing temperatures should

368 stabilize oscillations by weakening top-down effects (Vasseur \& McCann 2005; Binzer et al.

369 2016; Tabi et al. 2019). We found that temperature and nutrients interact to have divergent

370 impacts on population stability — with temperature stabilizing, and nutrients destabilizing

371 Euplotes sp. densities, but the opposite being true for T. pyriformis (Fig. 2h-i). We also observed

372 that top-down control of bacteria by both consumers was strongest when temperature and

373 nutrients were both high (and both low), while bottom-up effects were relatively invariant across

374 treatments (Fig. 4a, Fig. 5). Interestingly, these shifts in top-down control across environments

375 (Fig. 4a) coincided with significant changes in population stability that varied across trophic

376 levels (Fig. 2i). These results thus indicate that changes in the strength of top-down effects

377 between basal resources and consumers - instead of bottom-up effects — could be the dominant

378 mechanism through which higher temperature stabilize unstable fluctuations caused by nutrients.

Phenotypes mediate temperature and nutrients effects on food web dynamics

381 Our results indicate that phenotypic dynamics play a larger role in mediating environmental

382 impacts on food web dynamics than previously thought (Fig. 5, Appendix III Fig. S1). Previous

383 studies showed that predator-prey body size ratios significantly influence temperature and

384 nutrients effects (Binzer et al. 2012) and that body size responds to changes in nutrients, 
temperature, and ecological dynamics in specific ways (Tabi et al. 2019). Our results support-

386 but also extend - those results by showing that 1) phenotypic effects on ecological dynamics

387 may be stronger than the other way around, as also recently reported elsewhere (Gibert et al.

388 2021), 2) changes in prey size may more strongly drive changes in predator size than the other

389 way around, and 3) interactions involving phenotypic dynamics (eco-pheno, pheno-eco, or

390 pheno-pheno) vary in complex ways across environmental conditions, but seem to be generally

391 stronger at higher temperature (Fig. 4b-d, Fig. 5). Rapid phenotypic change has been suggested

392 as a main driver of food web rewiring in future climates (Barbour \& Gibert 2021), but little

393 experimental evidence exists. Here, we found that strong feedbacks between ecological and

394 phenotypic dynamics vary with temperature and nutrients, suggesting that rapid phenotypic

395 change influences food web responses to changing environmental conditions. Together, our

396 results emphasize the need to incorporate phenotypic dynamics in future studies of food web

397 responses to warming and eutrophication in a changing world.

ACKNOWLEDGMENTS

402 This work was supported by a U.S. Department of Energy, Office of Science, Office of

403 Biological and Environmental Research, Genomic Science Program Grant under Award Number

404 DE-SC0020362 to JPG. 


\section{Figures}
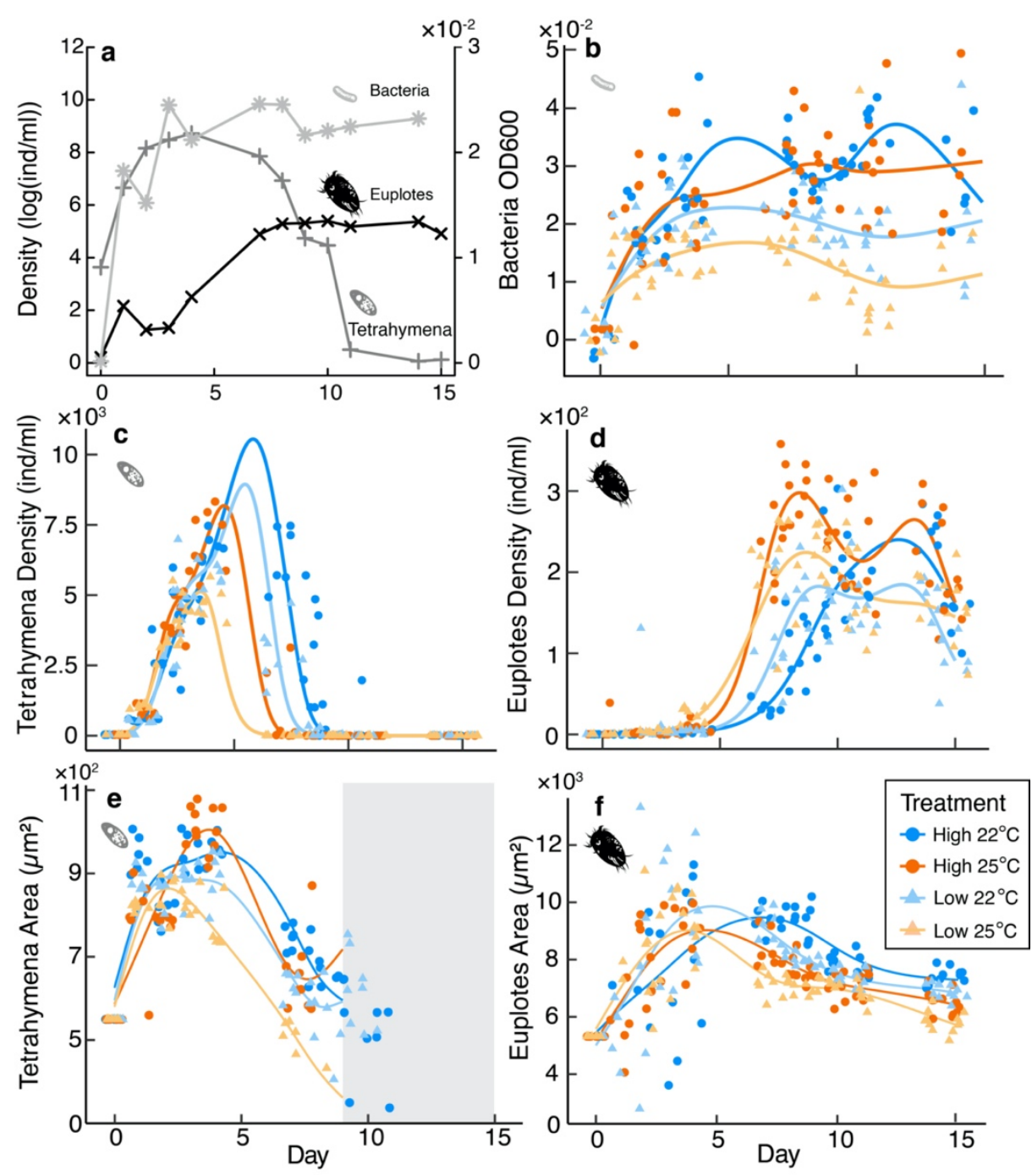

410 Figure 1. Population and phenotypic dynamics within experimental microcosms. (a) The average

411 population density across treatments shows the general population dynamics of each species. (b-

412 d) show GAMM smoothed population dynamics of bacteria, T. pyriformis and Euplotes sp.. (e-f)

413 show the changes in the area (body size) of both protists body over time. T. pyriformis were

414 effectively extinct by day 9. Greyed area indicates days for which data are insufficient to

415 estimate trait distributions. 

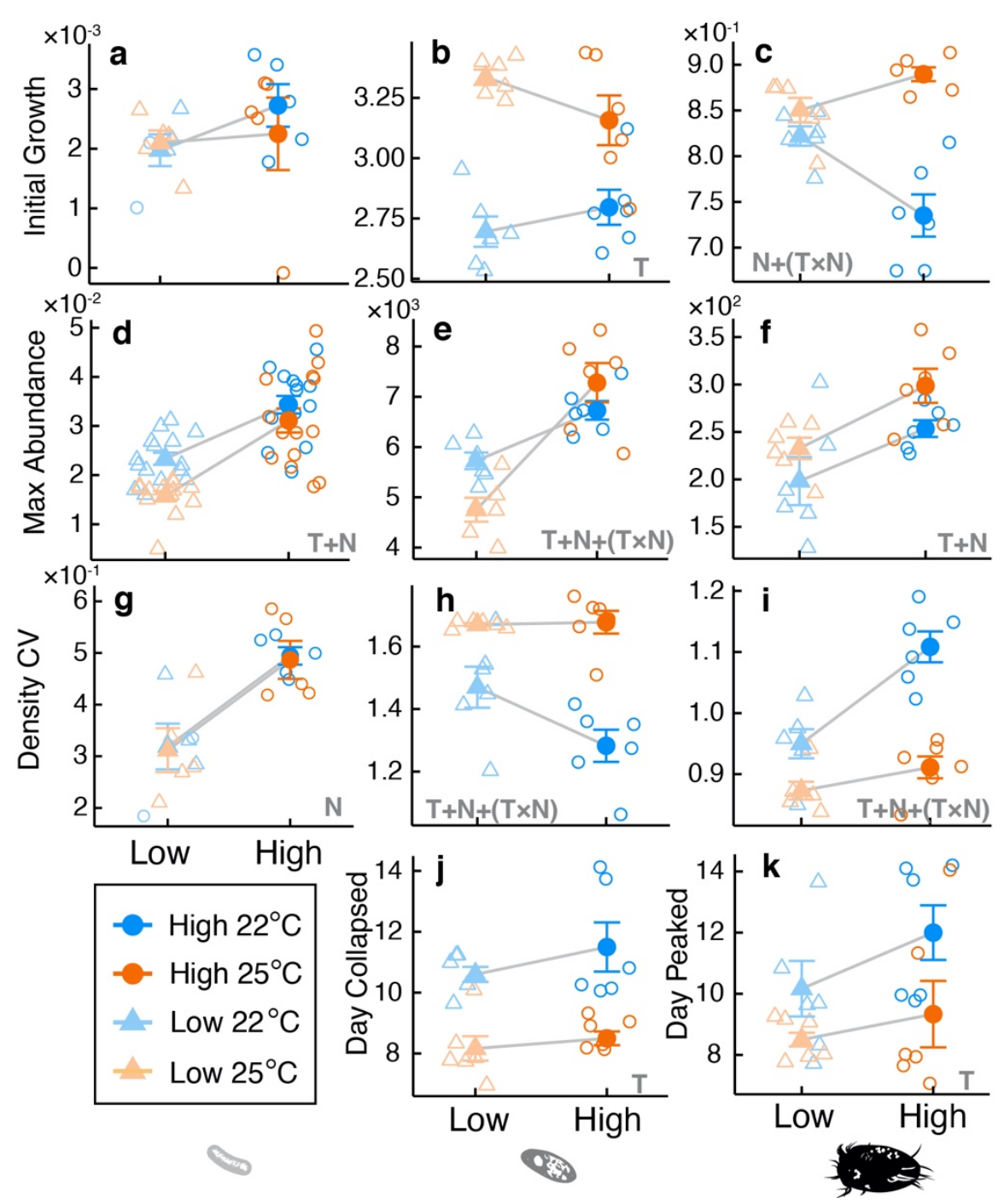

418 Figure 2. Interactive plot of effects of temperature and nutrients on descriptors of population

419 dynamics. Open circles and triangles represent data points from each replicate. Solid shapes are

420 the mean across 6 replicates. Grey letters $T, N$, and $(T \times N)$ represent statistically significant

421 effects from temperature, nutrients, and interactive effects between temperature and nutrients,

422 respectively. 


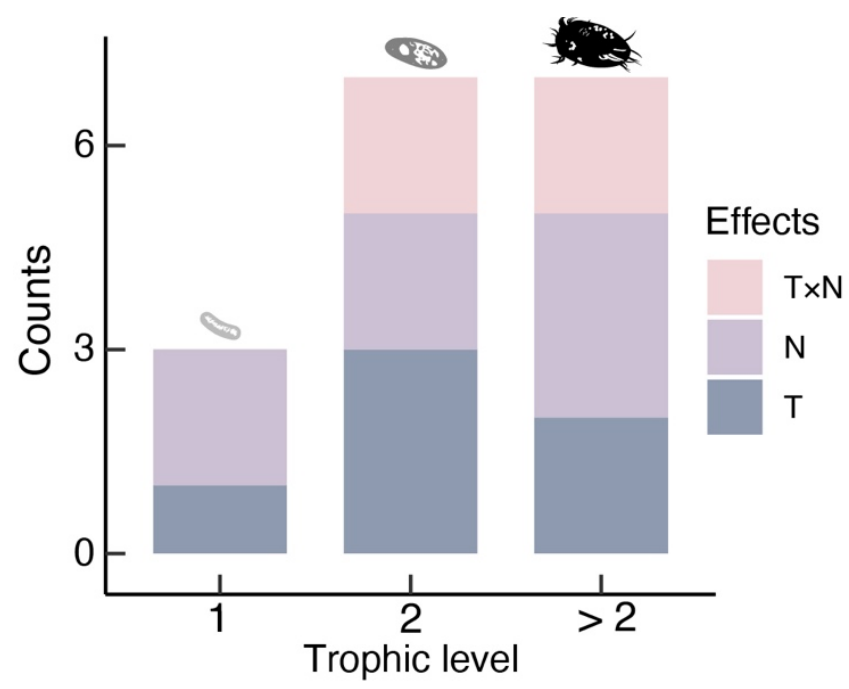

424 Fig. 3. Significant temperature and nutrients effects on ecological dynamics. T, N, and T $\times \mathrm{N}$

425 denote effects of temperature, nutrients, and interactive effects of temperature and nutrients,

426 respectively.

427 


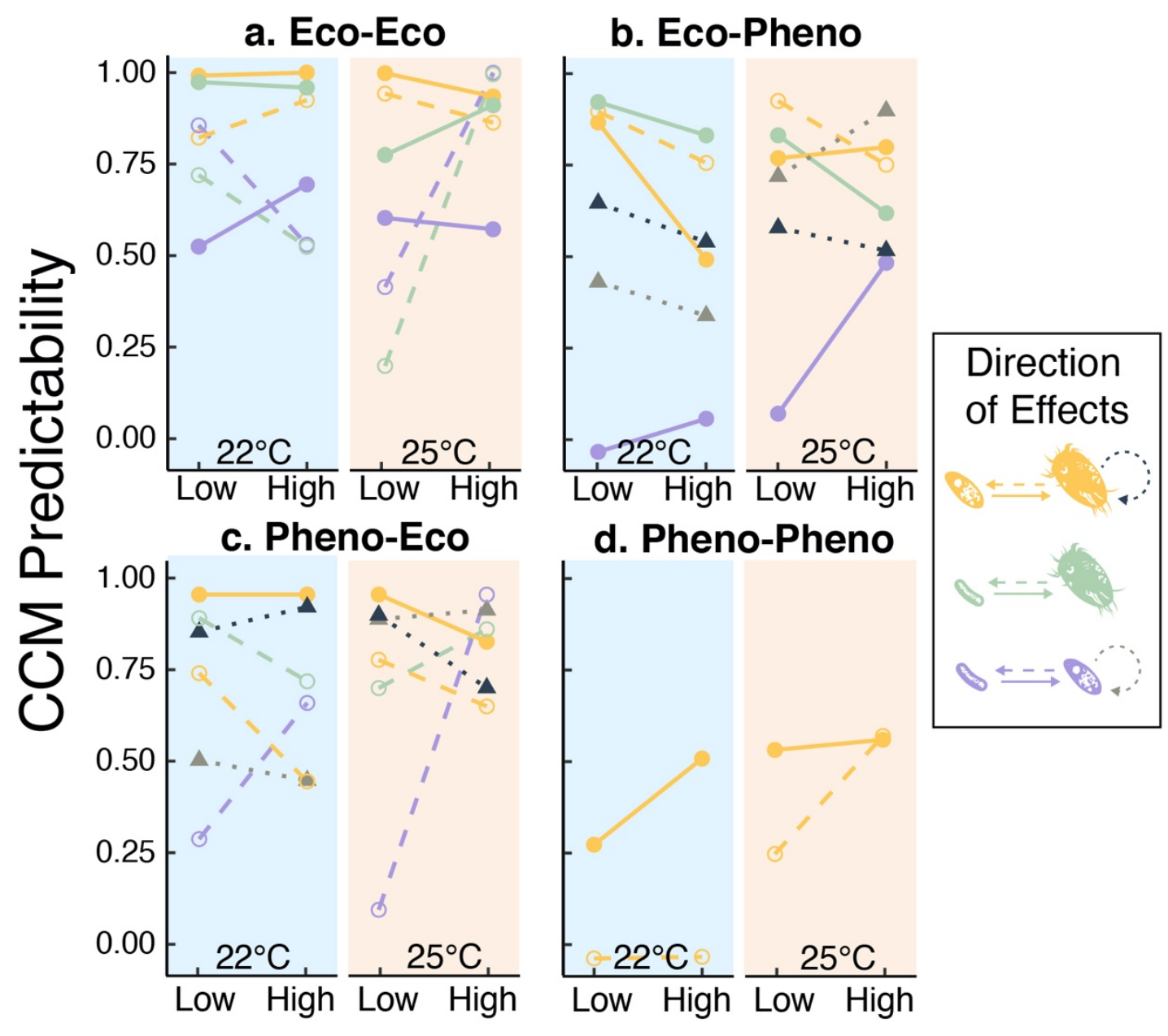

429 Figure 4. CCM predictabilities ecological and phenotypic dynamics across treatments for each

430 species pair. Circles represents CCM predictability of one species' effect on another species,

431 while the triangles representing intraspecific. Solid circles and solid lines represent CCM

432 predictability of bottom-up effects and open circles and dashed lines represent top-down effects.

433 Interactions with CCM predictability higher than 0.75 are considered to be strong interactions. 
a. Ecological effects

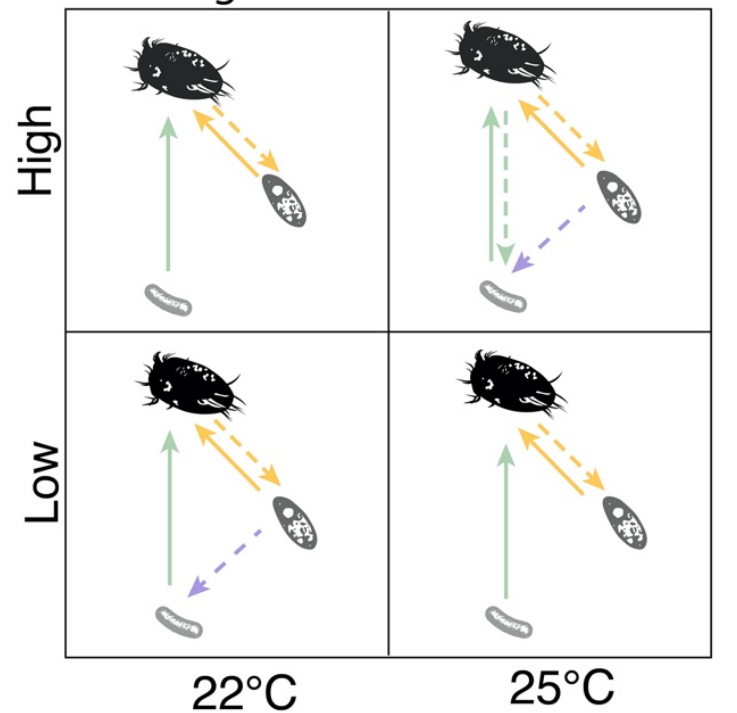

b. Ecological and phenotypic effects

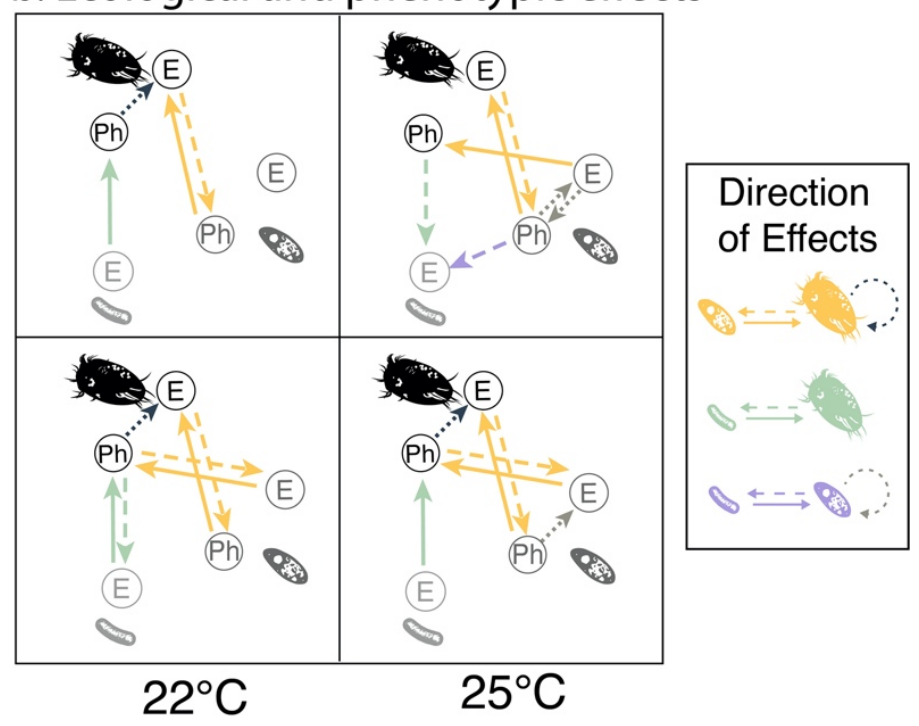

438 Figure 5. Strong effects (i.e., CCM predictability higher than 0.75) within food webs across

439 treatments. Panel (a) shows strong eco-eco effects (Fig. 4a). Panel (b) shows all strong

440 interactions between phenotypic $(\mathrm{Ph})$ and ecological $(\mathrm{E})$ dynamics (pheno-eco, eco-pheno and

441 pheno-pheno effects; Fig. 4b-d). Dashed and solid lines represent top-down and bottom-up

442 effects respectively. Dotted lines represent intraspecific effects. Color code is the same as in Fig.

$4434 a$. 
References:

Atkinson, D. (1995). Effects of temperature on the size of aquatic ectotherms: Exceptions to the general rule. J. Therm. Biol., 20, 61-74.

Atkinson, D., Ciotti, B.J. \& Montagnes, D.J.S. (2003). Protists decrease in size linearly with temperature: $c a .2 .5 \%{ }^{\circ} \mathrm{C}^{-1}$. Proc. R. Soc. Lond. B Biol. Sci., 270, 2605-2611.

Barbour, M.A. \& Gibert, J.P. (2021). Genetic and plastic rewiring of food webs under climate change. J. Anim. Ecol., 1365-2656.13541.

Barneche, D.R., Hulatt, C.J., Dossena, M., Padfield, D., Woodward, G., Trimmer, M., et al. (2021). Warming impairs trophic transfer efficiency in a long-term field experiment. Nature.

Bartley, T.J., McCann, K.S., Bieg, C., Cazelles, K., Granados, M., Guzzo, M.M., et al. (2019). Food web rewiring in a changing world. Nat. Ecol. Evol., 3, 345-354.

Barton, B.T., Beckerman, A.P. \& Schmitz, O.J. (2009). Climate warming strengthens indirect interactions in an old-field food web. Ecology, 90, 2346-2351.

Bernhardt, J.R., Sunday, J.M. \& O’Connor, M.I. (2018). Metabolic Theory and the TemperatureSize Rule Explain the Temperature Dependence of Population Carrying Capacity. Am. Nat., 192, 687-697.

Binzer, A., Guill, C., Brose, U. \& Rall, B.C. (2012). The dynamics of food chains under climate change and nutrient enrichment. Philos. Trans. R. Soc. B Biol. Sci., 367, 2935-2944.

Binzer, A., Guill, C., Rall, B.C. \& Brose, U. (2016). Interactive effects of warming, eutrophication and size structure: impacts on biodiversity and food-web structure. Glob. Change Biol., 22, 220-227.

Brose, U., Dunne, J.A., Montoya, J.M., Petchey, O.L., Schneider, F.D. \& Jacob, U. (2012). Climate change in size-structured ecosystems. Philos. Trans. R. Soc. B Biol. Sci., 367, 2903-2912.

Carlier, A., Riera, P., Amouroux, J.-M., Bodiou, J.-Y., Desmalades, M. \& Grémare, A. (2008). Food web structure of two Mediterranean lagoons under varying degree of eutrophication. J. Sea Res., 60, 264-275.

Clarke, A. (2006). Temperature and the metabolic theory of ecology. Funct. Ecol., 20, 405-412.

Dossena, M., Yvon-Durocher, G., Grey, J., Montoya, J.M., Perkins, D.M., Trimmer, M., et al. (2012). Warming alters community size structure and ecosystem functioning. Proc. $R$. Soc. B Biol. Sci., 279, 3011-3019.

Fenchel, T. (1974). Intrinsic rate of natural increase: The relationship with body size. Oecologia, $14,317-326$.

Ferenc, V. \& Sheppard, C.S. (2020). The stronger, the better - trait hierarchy is driving alien species interaction. Oikos, 129, 1455-1467.

Fussmann, G.F. (2000). Crossing the Hopf Bifurcation in a Live Predator-Prey System. Science, 290, 1358-1360.

Gauzens, B., Rall, B.C., Mendonça, V., Vinagre, C. \& Brose, U. (2020). Biodiversity of intertidal food webs in response to warming across latitudes. Nat. Clim. Change, 10, 264 269.

Gibert, J.P. (2019). Temperature directly and indirectly influences food web structure. Sci. Rep., 9, 5312.

Gibert, J.P., Han, Z.-Y., Wieczynski, D.J. \& Yammine, A. (2021). Rapid plastic shifts in body size precede and determine population growth. BioRxiv. doi: 10.1101/2021.07.15.452551 
Gounand, I., Kéfi, S., Mouquet, N. \& Gravel, D. (2016). Trait selection during food web assembly: the roles of interactions and temperature. Theor. Ecol., 9, 417-429.

Hautier, Y., Niklaus, P.A. \& Hector, A. (2009). Competition for Light Causes Plant Biodiversity Loss After Eutrophication. Science, 324, 636-638.

Hautier, Y., Seabloom, E.W., Borer, E.T., Adler, P.B., Harpole, W.S., Hillebrand, H., et al. (2014). Eutrophication weakens stabilizing effects of diversity in natural grasslands. Nature, 508, 521-525.

Irwin, A.J., Finkel, Z.V., Schofield, O.M.E. \& Falkowski, P.G. (2006). Scaling-up from nutrient physiology to the size-structure of phytoplankton communities. J. Plankton Res., 28, 459-471.

Joseph Park, Cameron Smith, George Sugihara and Ethan Deyle (2020). rEDM: Empirical Dynamic Modeling ('EDM'). R package version 1.5.0. https:/CRAN.Rproject.org $/$ package $=$ rEDM

Karl, T.R. (2003). Modern Global Climate Change. Science, 302, 1719-1723.

Kratina, P., Greig, H.S., Thompson, P.L., Carvalho-Pereira, T.S.A. \& Shurin, J.B. (2012). Warming modifies trophic cascades and eutrophication in experimental freshwater communities. Ecology, 93, 1421-1430.

Lee, G.H., Vonk, J.A., Verdonschot, R.C.M., Kraak, M.H.S., Verdonschot, P.F.M. \& Huisman, J. (2021). Eutrophication induces shifts in the trophic position of invertebrates in aquatic food webs. Ecology, 102.

Malcolm, J.R., Liu, C., Neilson, R.P., Hansen, L. \& Hannah, L. (2006). Global Warming and Extinctions of Endemic Species from Biodiversity Hotspots. Conserv. Biol., 20, 538-548.

Marañón, E., Cermeño, P., López-Sandoval, D.C., Rodríguez-Ramos, T., Sobrino, C., HueteOrtega, M., et al. (2013). Unimodal size scaling of phytoplankton growth and the size dependence of nutrient uptake and use. Ecol. Lett., 16, 371-379.

McCann, K.S. (2012). Food webs. Monographs in population biology. Princeton University Press, Princeton, N.J.

McClelland, J. \& Valiela, I. (1998). Changes in food web structure under the influence of increased anthropogenic nitrogen inputs to estuaries. Mar. Ecol. Prog. Ser., 168, 259271.

O’Connor, M.I. (2009). Warming strengthens an herbivore-plant interaction. Ecology, 90, 388398.

Petchey, O.L., McPhearson, P.T., Casey, T.M. \& Morin, P.J. (1999). Environmental warming alters food-web structure and ecosystem function. Nature, 402, 69-72.

R Core Team (2020). R: A language and for statistical computing. R Foundation for Statistical Computing, Vienna, Austria. URL https://www.R-project.org/.

Rip, J.M.K. \& McCann, K.S. (2011). Cross-ecosystem differences in stability and the principle of energy flux: Cross-ecosystem differences in stability. Ecol. Lett., 14, 733-740.

Rosenblatt, A.E. \& Schmitz, O.J. (2016). Climate Change, Nutrition, and Bottom-Up and TopDown Food Web Processes. Trends Ecol. Evol., 31, 965-975.

Rosenzweig, M.L. (1971). Paradox of Enrichment: Destabilization of Exploitation Ecosystems in Ecological Time. Science, 171, 385.

Savage, V.M., Gillooly, J.F., Brown, J.H., West, G.B. \& Charnov, E.L. (2004). Effects of Body Size and Temperature on Population Growth. Am. Nat., 163, 429-441. 
Sentis, A., Hemptinne, J.-L. \& Brodeur, J. (2014). Towards a mechanistic understanding of temperature and enrichment effects on species interaction strength, omnivory and foodweb structure. Ecol. Lett., 17, 785-793.

Shurin, J.B., Clasen, J.L., Greig, H.S., Kratina, P. \& Thompson, P.L. (2012). Warming shifts topdown and bottom-up control of pond food web structure and function. Philos. Trans. $R$. Soc. B Biol. Sci., 367, 3008-3017.

Sugihara, G., May, R., Ye, H., Hsieh, C. -h., Deyle, E., Fogarty, M., et al. (2012). Detecting Causality in Complex Ecosystems. Science, 338, 496-500.

Tabi, A., Petchey, O.L. \& Pennekamp, F. (2019). Warming reduces the effects of enrichment on stability and functioning across levels of organisation in an aquatic microbial ecosystem. Ecol. Lett., 22, 1061-1071.

Vandermeer, J. (2006). Omnivory and the stability of food webs. J. Theor. Biol., 238, 497-504. Vasseur, D.A. \& McCann, K.S. (2005). A Mechanistic Approach for Modeling TemperatureDependent Consumer-Resource Dynamics. Am. Nat., 166, 184-198.

Voigt, W., Perner, J., Davis, A.J., Eggers, T., Schumacher, J., Bährmann, R., et al. (2003). TROPHIC LEVELS ARE DIFFERENTIALLY SENSITIVE TO CLIMATE. Ecology, 84, 2444-2453.

Wang, J., Pan, F., Soininen, J., Heino, J. \& Shen, J. (2016). Nutrient enrichment modifies temperature-biodiversity relationships in large-scale field experiments. Nat. Commun., 7, 13960.

Wieczynski, D., Singla, P., Doan, A., Singleton, A., Han, Z., Yammine, A., Gibert, J.P. (2021). Simple traits predict complex temperature responses across scales. Research Square. doi:10.21203/rs.3.rs-116110/v1

Wood, S.N. (2011). Fast stable restricted maximum likelihood and marginal likelihood estimation of semiparametric generalized linear models: Estimation of Semiparametric Generalized Linear Models. J. R. Stat. Soc. Ser. B Stat. Methodol., 73, 3-36. 